\title{
An inventory of Canadian trauma systems: opportunities for improving access to trauma care
}

\author{
Christopher C.D. Evans, MD*; J.M. Tallon, MD, MSc ${ }^{\dagger}$; Jennifer Bridge, MPA, MHSc ${ }^{\ddagger}$; \\ Avery B. Nathens, MD, MPH, $\mathrm{PhD}^{\ddagger}$
}

\section{ABSTRACT}

Objective: Despite evidence that patients suffering major traumatic injuries have improved outcomes when cared for within an organized system, the extent of trauma system development in Canada is limited. We sought to compile a detailed inventory of trauma systems in Canada as a first step toward identifying opportunities for improving access to trauma care.

Methods: We distributed a nationwide online and mail survey to stakeholders intended to evaluate the extent of implementation of specific trauma system components. Targeted stakeholders included emergency physicians, trauma surgeons, trauma program medical directors and program managers, prehospital providers, and decision makers at the regional and provincial levels. A "snowball" approach was used to expand the sample base of the survey. Descriptive statistics were generated to quantify the nature and extent of trauma system development by region.

Results: The overall response rate was $38.7 \%$, and all levels of stakeholders and all provinces/territories were represented. All provinces were found to have designated trauma centres; however, only $60 \%$ were found to have been accredited within the past 10 years. Components present in $50 \%$ or fewer provinces included an inclusive trauma system model, interfacility transfer agreements, and a mechanism to track bed availability within the system.

Conclusion: There is significant variability in the extent of trauma system development in Canada. Although all provinces have designated trauma centres, opportunities exist in many systems to implement additional components to improve the inclusiveness of care. In future work, we intend to quantify the strength of the relationship between different trauma system components and access to definitive trauma care.

\section{RÉSUMÉ}

Objectif: Malgré l'existence de données probantes sur I'amélioration des résultats cliniques chez les patients ayant subi un trauma grave lorsqu'ils sont traités selon un système organisé de soins, l'élaboration de systèmes de traumatologie au Canada est peu étendue. Aussi avons-nous cherché à dresser un inventaire détaillé des systèmes de traumatologie au Canada comme première étape dans la recherche d'occasions d'améliorer l'accès aux soins dans ce contexte. Méthode: Un questionnaire d'enquête a été distribué en ligne et par la poste à différents intervenants, partout au pays, pour qu'ils évaluent l'étendue de la mise en œuvre de certains composants des systèmes de traumatologie. Les intervenants ciblés comprenaient les urgentologues, les chirurgiens traumatologues, les directeurs médicaux de programmes de traumatologie et les directeurs de programmes, les fournisseurs de soins préhospitaliers ainsi que les décideurs aux niveaux régional et provincial. Nous avons adopté la méthode en "boule de neige" afin d'élargir la base de l'échantillon visée par l'enquête. Des statistiques descriptives ont été générées pour permettre de quantifier, par région, la nature et l'étendue de l'élaboration des systèmes de traumatologie.

Résultats: Le taux de réponse global a atteint $38.7 \%$, et il comprenait des représentants de tous les niveaux d'intervenants ainsi que de toutes les provinces et de tous les territoires. Toutes les provinces ont des centres de traumatologie désignés, mais seulement $60 \%$ d'entre eux ont obtenu leur agrément au cours des 10 dernières années. Certains composants figuraient dans $50 \%$ ou moins des provinces, notamment un modèle de système de traumatologie "inclusif» ou ouvert, les ententes de mutation entre établissements et un mécanisme du suivi de la disponibilité des lits intégré au système.

From the *Department of Emergency Medicine, Kingston General Hospital and Hotel Dieu Hospital, Kingston, ON; †Departments of Emergency Medicine, Anesthesia, Surgery, and Community Health and Epidemiology, Dalhousie University, Halifax, NS; and fDepartment of Surgery, Sunnybrook Health Sciences Centre and St. Michael's Hospital, Keenan Research Centre in the Li Ka Shing Knowledge Institute, St. Michael's Hospital and Sunnybrook Research Institute, University of Toronto, Toronto, ON.

Correspondence to: Dr. Christopher C.D. Evans, Department of Emergency Medicine, Kingston General Hospital and Hotel Dieu Hospital, c/o 76 Stuart Street, Kingston, ON K7L 2V7; c.evans@queensu.ca.

This article has been peer reviewed. 
Conclusions: II existe des écarts importants en ce qui concerne l'étendue de l'élaboration des systèmes de traumatologie au Canada. Certes, toutes les provinces disposent de centres de traumatologie désignés, mais de nombreux systèmes gagneraient à inclure $d^{\prime}$ autres composants afin d'améliorer l'intégralité des soins. Ultérieurement, nous avons l'intention de quantifier la solidité de la relation entre différents composants des systèmes de traumatologie et l'accès à des soins spécialisés en traumatologie.

Keywords: emergency medical services, health care surveys, public health, trauma centres, wounds and injuries
In most countries, including Canada, injuries rank as the leading cause of death in the first four decades of life and cause more potential years of life lost than any illness. ${ }^{1-3}$ One of the most significant achievements in modern public health has been the development of trauma systems. Trauma systems integrate prehospital care, triage of patients to appropriate facilities for resuscitation and definitive care, rehabilitation, and injury prevention programs. ${ }^{4}$ As a public health intervention, trauma systems have been shown to reduce mortality from motor vehicle collisions by up to $17 \%{ }^{5,6}$ and improve functional outcomes in survivors of blunt trauma. ${ }^{7}$ A recent World Health Assembly resolution recognized the benefit of trauma and emergency care systems and emphasized the need for their integration in all nations. ${ }^{8}$

With Canada's population of approximately 34 million $^{9}$ and geographic expanse of nearly 10 million $\mathrm{km}^{2}{ }^{10}$ trauma systems are necessary to ensure that all citizens have prompt and reliable access to definitive trauma care. Previous research suggests that the state of trauma system development in Canada is highly variable, with many rural regions having very limited access to timely definitive trauma care. ${ }^{11}$ Unfortunately, for clinicians and administrators who wish to improve access to trauma care within their region, little evidence exists regarding the specific system components that should be optimized to achieve this goal. As the first step toward clarifying the relationships between trauma system components and access to care (and ultimately clinical outcomes), we sought to develop a detailed inventory of trauma systems in Canada. This study built on a previous survey of Canadian trauma systems by Hameed and colleagues in $2010^{11}$ and focused specifically on system components hypothesized to influence access to trauma care. The primary purpose of this study was to describe the prehospital triage and transfer practices, system organization, administrative structure, and interfacility transfer arrangements used in trauma systems across Canada.

\section{METHODS}

\section{Survey development}

This study was a cross-sectional, descriptive survey of trauma stakeholders in Canada. We developed a list of the essential components of a trauma system based on guidelines from the Trauma Association of Canada (TAC), ${ }^{4}$ the American College of Surgeons Committee on Trauma (ACS-COT) regional trauma systems consultation guide, ${ }^{12}$ and the US Health Resources and Services Administration Model Trauma System plan. ${ }^{13}$ By consensus, we narrowed this list of components to those that we believed most strongly influence access to acute trauma care. When consensus could not be reached on a component, it was included, and a final decision was made by the lead author after pilot testing the survey.

We focused on the model of trauma system designinclusive as opposed to exclusive. We evaluated system leadership through inquiries about the nature of the lead agency and the presence of a regional trauma medical director. We evaluated the presence of prehospital care protocols that would benefit critically injured patients, including field trauma triage criteria, ${ }^{14}$ trauma destination protocols, and the structured use of air medical transport. The types of acute care facilities within the system (designation status and last accreditation visit) were determined. The mechanisms for secondary triage were evaluated, including processes for identifying and transferring major trauma patients from community to regional trauma centres (e.g., interfacility transfer agreements and real-time tracking of bed availability). Participants were given the option to indicate whether each feature was present in their trauma system by choosing "yes," "no," or "unsure."

The final section of the survey gave participants the opportunity to provide narrative comments on the features of their trauma system that they felt either facilitated or impeded access to care. A separate box could be "clicked" to indicate consent for a follow-up telephone interview with one of the authors. Participants 
who provided such consent were contacted either to clarify their survey responses or provide details of their trauma system.

The survey was pilot-tested by three physicians with expertise in trauma system design. Following this, a revised final version was developed in both written and electronic formats.

\section{Respondents}

Potential respondents were identified using the TAC database of Canadian trauma centres, provincial ministry of health websites, and regional trauma program websites and included clinicians, administrators, and policy makers who were considered stakeholders in their respective trauma system. We used a "snowball" sampling technique ${ }^{15}$ in which respondents were asked to identify other trauma system stakeholders, and when appropriate, these individuals were invited to complete the survey. No remuneration or other incentive was provided for participation.

\section{Survey distribution}

From May to August 2011, potential subjects were sent an email notification that they had been selected for invitation to participate in the study and that the survey would be delivered within 2 weeks. Participants had a choice of three completion methods: 1) following a hyperlink to complete an online version of the survey (Delvinia Interactive Inc., Toronto, $\mathrm{ON}), 2$ ) requesting a paper version of the survey to be mailed to them, or 3) survey completion by telephone with an interviewer. Two reminder emails were sent at 2-week intervals for invitees who did not initially complete the survey. Each of the emails above included a letter of information about the study and contact information for the principal investigator should the participant wish to opt out. Participants indicated via a check box at the start of the survey that they read the letter of information, agreed to participate, and consented to their responses being used for research purposes.

\section{Data analysis}

Survey results were compiled in a password-protected electronic spreadsheet. Given that definitive trauma care for the three Canadian territories is provided by the provinces of Alberta, British Columbia, and Manitoba, we grouped each territory with the province responsible for its trauma coverage. Throughout the text, we have used the term province for simplicity, recognizing that in these three aforementioned cases, we are referring to a province/ territory combination.

For survey questions where responses regarding the presence or absence of specific trauma system components were inconsistent, we defined whether the component was present, absent, or unclear based on the majority of responses, and whenever possible, we confirmed the accuracy of our categorization using publicly available information on either government websites or the TAC homepage (<http://www.traumacanada.org $>$ ).

The study received approval from the Research Ethics Board of St. Michael's Hospital.

\section{RESULTS}

Ninety-three surveys were distributed, and 36 were completed, yielding a response rate of $39 \%$. Participants represented a range of stakeholders, including 9 emergency medical services personnel (27\%), 9 regional or provincial government representatives (27\%), 7 trauma program coordinators/managers (22\%), 7 trauma program medical directors (19\%), 3 emergency physicians or trauma surgeons $(8 \%)$, and 1 trauma researcher $(3 \%)$. Some of the preceding participants were categorized in more than one group. To clarify survey responses and enhance accuracy, we conducted follow-up interviews with respondents from British Columbia, Saskatchewan, and Manitoba. These provinces were singled out for this follow-up because there were some conflicting responses from participants in these regions that could not be clarified using online sources.

Table 1 provides a description of the survey respondents by province. Response rates by stakeholder type are provided in Table 2. The highest response rates were from regional or provincial health administrators $(56 \%)$, whereas the lowest response rates $(18 \%)$ were from emergency physicians and trauma surgeons.

A summary and definitions of the key components of a trauma system that were evaluated in the survey are presented in Table 3. These tables indicate that five provinces have an inclusive system design (British Columbia and Yukon Territories, Alberta and 


\begin{tabular}{|lccc|}
\hline \multicolumn{4}{|l|}{ Table 1. Survey response rates by province or province/territory } \\
\hline Province or territory & Sent & Responses & Response rate (\%) \\
\hline Prince Edward Island & 1 & 1 & 100 \\
Nova Scotia & 5 & 3 & 60 \\
Alberta and Northwest Territories & 20 & 10 & 50 \\
New Brunswick & 5 & 2 & 40 \\
Ontario & 33 & 13 & 39 \\
British Columbia and Yukon & 6 & 2 & 33 \\
Manitoba and Nunavut & 8 & 2 & 25 \\
Quebec & 4 & 1 & 25 \\
Newfoundland and Labrador & 4 & 1 & 25 \\
Saskatchewan & 7 & 1 & 14 \\
Total & 93 & 36 & 39 \\
\hline Territories are grouped with provinces responsible for definitive trauma care. & & \\
\hline
\end{tabular}

Northwest Territories, Quebec, Nova Scotia, and New Brunswick), whereas the remainder follow a more exclusive system model. Only two provinces (Manitoba, Newfoundland and Labrador) had no lead agency identified. All regions had centres designated to receive trauma patients; however, only $60 \%$ were found to have been accredited within the past 10 years. A trauma system plan, regional trauma medical director, and advisory committee were present in most provinces. Provinces with an inclusive system were more likely to have accredited trauma centres, a system plan, a lead agency, a medical director, and a trauma system advisory committee.

Emergency medical services leadership was present across all regions, and most had protocols in place to identify and transport critically injured patient to an appropriate centre. Interfacility transfer agreements and real-time tracking of bed availability within the system were incorporated into relatively fewer systems.

\section{DISCUSSION}

It is clear that trauma systems improve survival and functional outcomes for critically injured patients..$^{5-7,16}$
One of the mechanisms for this is through the provision of prompt access to definitive care at a trauma centre. ${ }^{17}$ In Canada, we face challenges in ensuring access to trauma care for citizens living outside urban regions because of our geographic expanse, sparse population densities, limited road access, and inclement weather. ${ }^{11} \mathrm{~A}$ recent study of Canadian trauma systems indicated that $78 \%$ of Canadians live within a 1-hour travel time of a level I or II trauma centre. However, marked disparities exist between provinces, with only $40 \%$ of residents in some provinces falling within this catchment zone. ${ }^{11}$

We found that considerable variation exists in the design and maturity of trauma systems across Canada. Five provinces appear to follow the "inclusive" trauma system model. Provinces including British Columbia, Alberta, Quebec, and New Brunswick, which designate many, if not all, acute care hospitals within their province on a spectrum from level I (tertiary care centre) to level V (small rural hospital) according to their resource availability, as defined in the TAC guidelines, ${ }^{4}$ represent examples of inclusive trauma systems. Other regions, such as Ontario, Saskatchewan, and Manitoba, have developed more "exclusive" system models, which focus

\begin{tabular}{|lcr|}
\hline Table 2. Survey response rates by stakeholder type & \\
\hline Stakeholder type & Sent, $n(\%)$ & Responses, $n(\%)$ \\
\hline Regional or provincial health administrator & $19(20)$ & $9(56)$ \\
Trauma program manager & $14(15)$ & $7(50)$ \\
Trauma researcher & $2(2)$ & $1(50)$ \\
Emergency medical services & $19(20)$ & $9(47)$ \\
Trauma medical director & $25(27)$ & $7(28)$ \\
Emergency physician or trauma surgeon & $17(18)$ & $3(18)$ \\
\hline
\end{tabular}




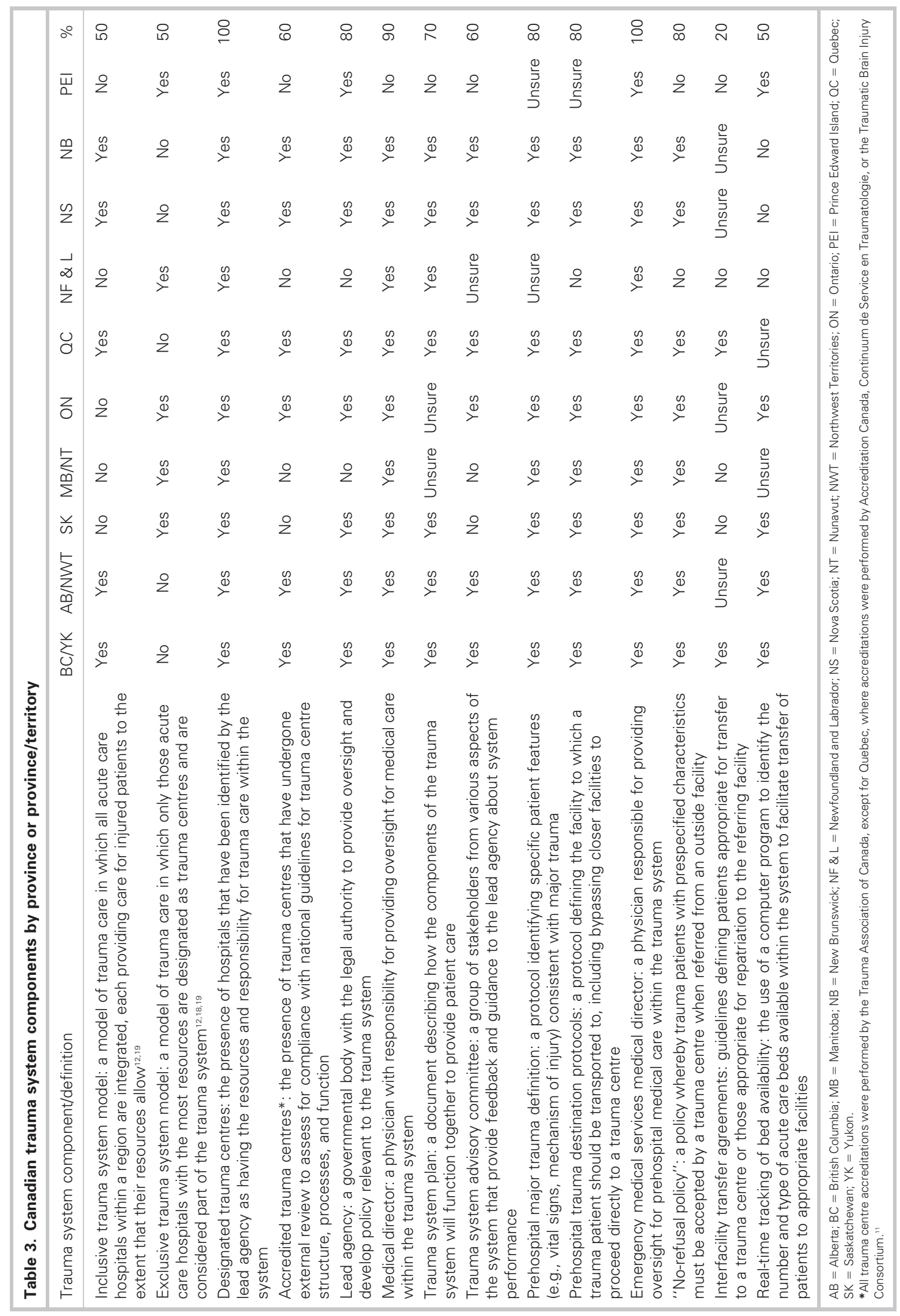


on the care provided at designated level I trauma facilities and place less emphasis on the integration of other acute care facilities within the system. ${ }^{12,18,19}$

In the United States, states with an inclusive trauma system model have been found to have significantly lower mortality than those with exclusive trauma system models. ${ }^{18}$ Exactly how inclusive systems improve mortality is not entirely clear; however, improved prehospital triage, better resuscitative care at rural hospitals, more efficient secondary triage processes, stronger system leadership, or other, yet unidentified factors may be responsible. ${ }^{18}$ Our results indicate that provinces with inclusive trauma system models were more likely to have accredited trauma centres, a lead agency, a system plan, and a system advisory committee. It is likely that in some regions of Canada, an increased emphasis on inclusive models of trauma care could improve outcomes.

All Canadian trauma systems included designated trauma centres; however, only $60 \%$ of provinces have had these centres accredited within the past 10 years. Given evidence that the accreditation process improves outcomes at trauma centres, ${ }^{20,21}$ ongoing efforts to ensure trauma centre accreditation should be encouraged within all trauma systems.

An identifiable lead agency with ultimate responsibility for the trauma system and a system plan, which delineates the responsibilities of all centres and providers within the system, was present in eight and seven provinces, respectively. Although there is no direct evidence that such components improve access to care or clinical outcomes, these components have been identified as "essential elements" of a trauma system by a number of groups, including the $\mathrm{TAC}^{4}$ and the ACS-COT, ${ }^{12}$ and could be considered priority improvement items in systems currently lacking them.

Prehospital triage is a critical component of trauma system design. ${ }^{14}$ In Ontario, patients with comparable injuries from motor vehicle collisions who were triaged directly to a trauma centre were found to have a $30 \%$ lower 48-hour mortality than those who were initially triaged to a nontrauma centre. ${ }^{22}$ Prehospital trauma care systems appear to be relatively well organized throughout Canada, with most regions having prehospital protocols that define a major trauma patient, address triage, and provide guidance regarding appropriate hospital destination.

However, in many systems, there appear to be opportunities to improve the processes of transferring trauma patients from the initial hospital of care to a trauma centre. Only 20\% of trauma systems have transfer agreements between referring and definitive care facilities, and only $50 \%$ of systems track real-time bed availability within the region or province. An improvement in secondary triage processes throughout the country is likely to improve the timeliness of access to definitive trauma care for Canadians living outside urban regions.

There are limitations to our study. First, our overall survey response rate was only $39 \%$, despite efforts to increase the response rate, including the snowball sampling technique, email reminders, and options to complete the survey using electronic, written, or telephone formats. It is likely that a factor in our low response rate relates to our approach of inviting multiple respondents from the same trauma program. In many cases, one individual completed the survey on behalf of the program, thus reducing the collective response rate but still maintaining the descriptive objective of our study design. Despite these limitations, our response rate is consistent with that of other national surveys involving physicians. ${ }^{23}$

An additional challenge was obtaining responses from all regions of the country. We used a robust sampling strategy, sought guidance from national trauma experts, and thoroughly searched provincial government websites. However, in Saskatchewan, Manitoba, Quebec, Newfoundland and Labrador, and Prince Edward Island, it was very difficult to identify trauma system stakeholders to invite for participation. We cannot exclude the possibility that some participants were missed in the recruitment phase of the study but feel it is likely that our challenges in this regard in some locations are a reflection of the developing state of the trauma systems involved and the resulting absence of an identifiable individual with system leadership responsibilities. Despite this, we were able to obtain responses from a range of stakeholders from all provinces in Canada.

\section{CONCLUSION}

This study provides a comprehensive overview of trauma systems in Canada. Although all areas have designated trauma centres, in many locations, opportunities appear to exist to improve access to trauma care by increasing system inclusiveness and improving patient transfer from community hospitals to trauma centres. We hope our results will encourage comparison and benchmarking between provinces and stimulate policy changes to improve access to trauma care. 
In future work, we intend to quantify the strength of the relationship between different trauma system components and access to definitive trauma care.

Competing interests: None declared.

\section{REFERENCES}

1. Canadian Institute for Health Information. National Trauma Registry 2011 report: hospitalizations for major injury in Canada, 2008-2009 data. Ottawa: Canadian Institute for Health Information; 2011.

2. Peden M, McGee K, Krug EG. Injury: a leading cause of the global burden of disease. Geneva: World Health Organization; 2000.

3. Peden M, McGee K, Sharma G. The injury chart book: a graphical overview of the global burden of injuries. Geneva: World Health Organization; 2002.

4. Trauma Association of Canada. Trauma system accreditation guidelines: 4th edition, 2011. Available at: http://www. traumacanada.ca/accreditation_committee/Accreditation_ Guidelines_2011.pdf (accessed July 26, 2012).

5. Nathens AB, Jurkovich GJ, Cummings P, et al. The effect of organized systems of trauma care on motor vehicle crash mortality. FAMA 2000;283:1990-4.

6. Nathens AB, Jurkovich GJ, Rivara FP, et al. Effectiveness of state trauma systems in reducing injury-related mortality: a national evaluation. 7 Trauma 2000;48:25-30.

7. Gabbe BJ, Biostat GD, Simpson PM, et al. Improved functional outcomes for major trauma patients in a regionalized, inclusive trauma system. Ann Surg 2012;255: 1009-15.

8. World Health Assembly. Health systems: emergency care systems - report by the Secretariat, 2012. Available at: http:// www.who.int/gb/ebwha/pdf_files/WHA60/A60_21-en.pdf (accessed July 26, 2012).

9. Statistics Canada. Canada at a glance 2012-population, 2012. Available at: http://www.statcan.gc.ca/pub/12-581-x/2012000/ pop-eng.htm (accessed July 26, 2012).

10. Statistics Canada. Human activity and the environment: annual statistics: section 2: annual statistics: Canada's physical environment, 2012. Available at: http://www.statcan.gc.ca/pub/ 16-201-x/2009000/part-partie2-eng.htm (accessed July 26, 2012).
11. Hameed SM, Schuurman N, Razek T, et al. Access to trauma systems in Canada. 7 Trauma 2010;69:1350-61.

12. Ball J, Burton RA, Channarayapatn S, et al. Regional trauma systems: optimal elements, integration, and assessment-American College of Surgeons Committee on Trauma: systems consultation guide. Chicago: American College of Surgeons; 2008.

13. Health Resources Service Administration. Model trauma system planning and evaluation. Washington (DC): Department of Health and Human Services; 2006.

14. Centers for Disease Control and Prevention. Guidelines for field triage of injured patients: recommendations of the National Expert Panel on Field Triage. MMWR Morb Mortal Wkly Rep, 2008;57(No. RR-1);1-35.

15. Graham ID, Logan J, O'Connor A, et al. A qualitative study of physicians' perceptions of three decision aids. Patient Educ Couns 2003;50:279-83.

16. Tallon JM, Fell DB, Karim SA, et al. Influence of a province-wide trauma system on motor vehicle collision process of trauma care and mortality: a 10-year follow-up evaluation. Can 7 Surg 2012;55:8-14.

17. MacKenzie EJ, Rivara FP, Jurkovich GJ, et al. A national evaluation of the effect of trauma-center care on mortality. $N$ Engl 7 Med 2006;354:366-78.

18. Utter GH, Maier RV, Rivara FP, et al. Inclusive trauma systems: do they improve triage or outcomes of the severely injured? 7 Trauma 2006;60:529-35.

19. Sasser SM, Hunt RC, Sullivent EE, et al. Guidelines for field triage of injured patients. Recommendations of the National Expert Panel on Field Triage. MMWR Recomm Rep 2009;23: $1-35$.

20. Pasquale MD, Peitzman AB, Bednarski J, et al. Outcome analysis of Pennsylvania trauma centers: factors predictive of nonsurvival in seriously injured patients. 7 Trauma 2001;50: 465-72.

21. Simons R, Kasic S, Kirkpatrick A, et al. Relative importance of designation and accreditation of trauma centers during evolution of a regional trauma system. 7 Trauma 2002;52: 827-33.

22. Haas B, Stukel TA, Gomez D, et al. The mortality benefit of direct trauma center transport in a regional trauma system: a population-based analysis. 7 Trauma Acute Care Surg 2012; 72:1510-7.

23. Grava-Gubins I, Scott S. Survey response rates among Canadian physicians and physicians-in-training. Can Fam Physician 2008;54:1424-30. 